\title{
Etude sémiotique et sémantique de l'espace \\ dans Notre Dame \\ de Paris de Victor Hugo.
}

\author{
Salwa Hussein Abd El Moneim \\ Professeur adjoint \\ Faculté des Lettres \\ Université de Helwan
}


$-11 \cdot-$

PDF created with pdfFactory Pro trial version www.pdffactory.com 


\section{Résumé : \\ Etude sémiotique et sémantique de l'espace dans Notre Dame de Paris de Victor Hugo.}

Notre Dame de Paris de Victor Hugo fait à la fois partie des chefs d'œuvre du patrimoine littúr ai re fran çais et mondial. II s'agit d'un roman historique, genre plus ou moins inspiré des travaux de l'écrivain écossais Wolter Scott @e genre $p$ end, pour toile de fond de la narration, un événeme nt réel ar un épi sode historique bien défii.l I mê le d es $p$ ersonnages e $t$ des événements réels à d'autres fuitifs et dével oppe une i ntrigre ayant un aspect vraisemblable.

Dans son ouvrage, Hugo situe l'action dans un cadre bi en précis, celui du Paris médiéval. Toutefois, loin de s'attarder sur l'étude de l'espace dans sa dimension esthétique et hi storique riche mais énormément abordée, nous nous intéresserons, dans ce travail, à étudier l'espace dans une optique muvel le à savoir l'optique sém dique et séman tique

Nous avons bien remarqué que les diverses indications spatil es que renfer ne l e rama $\mathrm{n}$ sont porteuses de sens et intrie me nt l iées aux événeme nt $s$ qui s'y dér aul ent. L' es pace non seulement situe l'action ma is I'amp l ifé, la sugg ère, la reproduit, la reflète et la concrétie. II s' gi t d' un actant aus si effice que I eper sonnage du ranan. L'occ upationde I 'esace sur le plan horizontal ou vertical a lú aus si un as pect si gi fart non négligeable.

L'espace est non seulement un simple décor situant l'action dans un no yen âge ló it ai n ma i sils' gi t d' un langage 
bien élaboré et d'un mode d'expression porteur de concepts sémantíues $\mathrm{m} I$ ṫ̉les Nus essayerons, à travers ce travail, de souligner comment l'espace reflète la fition et lar eff orce tat en reproduisant les traits de caractère principaux des personnages l'occupant et comment le déplacement des personnages répond à des transformations qu' ils s ubi ssent. Pour le faire, nous nous référons aux travaux d'Youri Lotman, de Marie Laure Ryan ainsi qu'à ceux d'autres théoriciens.

\section{Mots clefs:}

Espace sémantio- sém diqu - Qc upationde I' espace relations spatiales bi ná res - le rapport haut/bas - le rapport proche/lointain - espace métaphorique. 


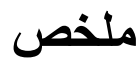

دراسة دلالات و سيميائية المكان

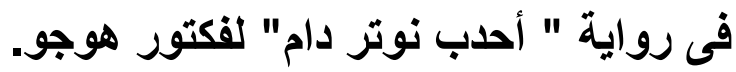

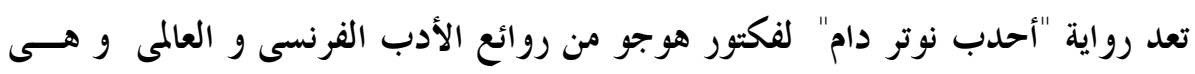

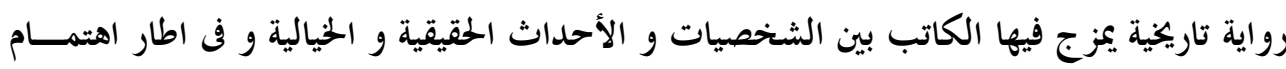

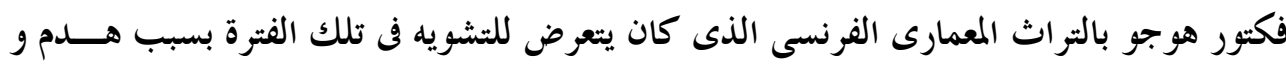

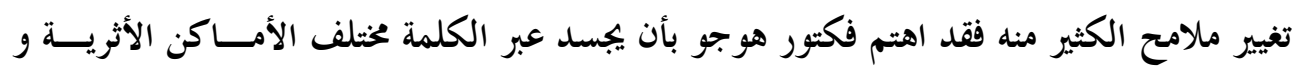

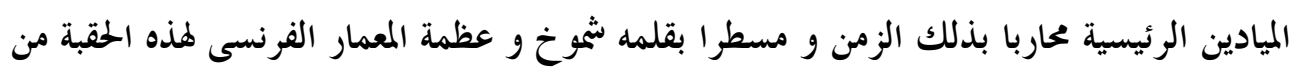

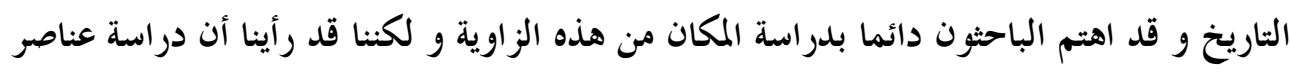

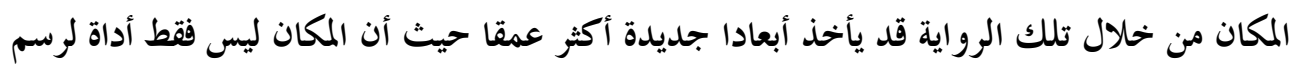
صورة عاصمة النور فى العصور الوسطى و لكن الكاتب قد استغل دلالات المكان المختلفة سواء منها الايحائى أو اللفظى للتعبير عن مضمون الرواية و عن مختلف الأحداث التى تدور بها و الشخصيات التى تظهر فيها.

فالمكان بدلالاته و ايحاءاته السيميائية و اللفظية و البجازية قد تحول الم أداة فاعلة للتـأثير و

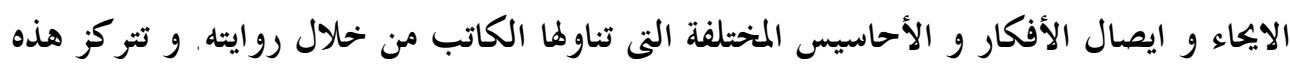

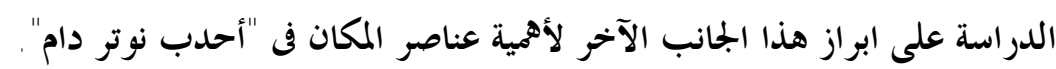




\section{1- Introduction, aspect théorique et formulation de la problématique:}

Notre Dame de Paris de Victor Hugo fait à la fois partie des chefs d'œuvre du patrimoine littéraire français et mondial. L'œuvre a été étudiée et abordée de divers points de vue. Elle a été représentée sur scène et adaptée cinématographiquement et a inspiré des milliers d'autres divertissements. Il s'agit d'un roman historique, genre plus ou moins inspiré des travaux de l'écrivain écossais Wolter Scott. Ce genre prend, pour toile de fond de la narration, un événement réel ou un épisode historique bien défini. Il mêle des personnages et des événements réels à d'autres fictifs et développe une intrigue ayant un aspect vraisemblable.

Dans son ouvrage, Hugo situe l'action dans un cadre bien précis, celui du Paris médiéval. Défenseur du patrimoine architectural français menacé de disparition et d'oubli dans quelques cas, de modification sinon de destruction dans d'autres cas, Hugo insiste à faire dans son ouvrage un tableau plus ou moins exhaustif de la capitale parisienne de jadis. Comme dans les autres romans du genre, l'auteur recourt à une ample documentation et cite ses sources aux lecteurs. En fait, ce tableau pittoresque et détaillé, tout en donnant à l'ouvrage une nouvelle dimension, constitue un élément de ralentissement de l'action par 
de longues digressions descriptives et topographiques. Qu'elles soient jugées positivement ou négativement, ces digressions amplifient l'aspect documentaire et esthétique de l'ouvrage.

Toutefois, loin de s'attarder sur l'étude de l'espace dans sa dimension esthétique et historique riche mais énormément abordée, nous nous intéresserons, dans ce travail, à étudier l'espace dans une optique nouvelle à savoir l'optique sémiotique et sémantique.

Nous avons bien remarqué que les diverses indications spatiales que renferme le roman sont porteuses de sens et intimement liées aux événements qui s'y déroulent. L'espace non seulement situe l'action mais l'amplifie, la suggère, la reproduit, la reflète et la concrétise. Il s'agit d'un actant aussi efficace que le personnage du roman. L'occupation de l'espace sur le plan horizontal ou vertical a lui aussi un aspect signifiant non négligeable. L'espace est non seulement un simple décor situant l'action dans un moyen âge lointain mais il s'agit d'un langage bien élaboré et d'un mode d'expression porteur de concepts sémiotiques multiples. Nous essayerons donc, à travers cette étude, de souligner comment l'espace reflète la fiction et la renforce tout en reproduisant les traits de caractère principaux 
des personnages l'occupant et comment le déplacement des personnages répond à des transformations qu'ils subissent.

Pour le faire, nous nous référons aux travaux d'Youri Lotman, de Marie Laure Ryan et de M. Issacharoff ${ }^{1}$.

D'après Youri Lotman, "les concepts haut/bas, droit/gauche, proche/lointain, ouvert/fermé, délimitél nondélimité, discret/continue se trouvent être un matériau pour construire des modèles culturels sans aucun contenu spatial et ils prennent le sens de valable/non-valable, bon/mauvais, les siens/les étrangers, accessible/inaccessible, mortel/immortel, etc" (Y. Lotman, 1973: 313)

Lotman s'intéresse donc aux relations spatiales binaires qui, selon lui, reproduisent des rapports qu'on peut qualifier de "non spatiaux" voire même de "métaphoriques".

Nous développons de plus en plus son idée en se référant à ki-Jeong Song, de l'Université d'Ewha à Séoul qui dit que :

De la différence entre les espaces opposés, il se produit un phénomène sémiotique. (...) Or l'espace n'a pas de sens propre en lui-même. Ce qui est important, ce n'est pas le signe lui-même, mais la relation entre les signes.

$(2012: 374)$

Pour la théoricienne Marie Laure Ryan, elle conçoit les choses différemment et prend un cheminement tout-à-fait opposé. Elle 
part des concepts métaphoriques que renferme l'espace pour illustrer les valeurs sémantiques du texte artistique.

When the notion of space refers to a formal pattern, it is taken in a metaphorical sense, since it is not a system of dimensions that determines physical position, but a network of analogical or oppositional relations perceived by the mind. It is the synchronic perspective necessitated for the perception of these designs and the tendency to associate the synchronic with the spatial that categorizes them as spatial phenomena ${ }^{2}$. (M.-L Ryan, 2009)

Partons de ces concepts et revenons à l'œuvre de Victor Hugo, nous remarquons, dès le début, le grand intérêt que l'auteur accorde aux lieux étant donné que le titre de l'ouvrage glorifie l'espace emblématique du roman à savoir la cathédrale de NotreDame de Paris. Outre le titre, certains autres chapitres portent le nom de divers autres lieux médiévaux riches de connotations. Le texte lui-même est parsemé d'indications spatiales comme on le verra à travers ce travail.

\section{Etude des relations spatiales binaires:}

Pour les relations spatiales déjà évoquées par Lotman, nous allons, dans cette première partie du travail, essayer d'examiner les aspects et les fonctions des espaces supposés renfermer une relation binaire pour voir leur degré d'efficacité suggestive par rapport au contenu conceptuel du texte. 


\subsection{Etude de la relation spatiale relevant du concept haut/bas:}

Prenons tout d'abord le concept haut/ bas qui nous paraît reproducteur de sens dans ce roman. Nous allons essayer de répartir les lieux fréquentés par les personnages en plusieurs catégories par rapport au concept haut/bas.

Dans un premier temps, on a pu distinguer plusieurs niveaux à savoir :

- le haut, le trop haut, d'un côté et le bas et le trop bas d'un autre.

Si le haut, comme sa connotation l'indique, révèle la transcendance, la spiritualité, l'élévation, la grandeur et le sacré; par contre, le trop haut, par l'excès de hauteur, dégénère du positif vers le négatif comme on le montrera plus loin. De même, si le bas revêt un aspect négatif, le trop bas est encore pire et revêt un aspect amer et dégoutant.

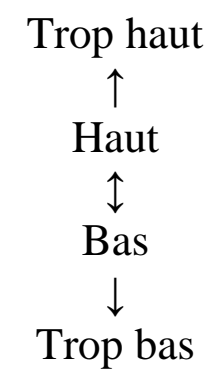

Dans Notre Dame de Paris, le trop haut est surtout occupé par deux personnages fictifs et un personnage réel à savoir 
l'archidiacre Claude Frollo et Quasimodo, d'un côté, et le roi Louis XI de l'autre.

Pour se rendre dans la cellule de son frère l'archidiacre, Jehan a dû "monter la vis de Saint-Gilles qui mène aux étages supérieurs de la tour". Mais arrivant sur la galerie des colonnettes, il a dû reprendre "son ascension" et c'est ainsi qu'il accède à la cellule secrète de son frère. C'est toujours de cette cellule que le prêtre voyait d'en haut tout Paris:

"Tout Paris était sous ses pieds" (V. Hugo, 2006: 336)

C'est toujours de cette tour qu'il guettait les déplacements de la jeune tsigane:

"(...) Mais dans toute cette ville, l'archidiacre ne regardait qu'un point du pavé, la place du Parvis ; dans toute cette foule, qu'une figure, la bohémienne " (Hugo, 2006 : 336)

C'est là où on le trouve souvent :

"Tous les jours, une heure avant le coucher du soleil, l'archidiacre montait l'escalier de la tour, et s'enfermait dans cette cellule, où il passait quelquefois des nuits entières." (Hugo, 2006 : 335)

Si pour Claude Frollo, il s'agit d'un homme de qualité, pour qui l'ascension semble répondre à la condition sociale et à la vénération dont il jouit dans son entourage surtout dans un moyen âge encore soumis au joug de l'église, il faut bien noter que la description de la cellule qu'il occupe revêt un aspect 
sombre et lugubre. Des sémantismes à valeur négative directe et indirecte semblent être utilisés pour décrire cette pièce à la fois "sombre", "à peine éclairé(e)", " têtes de mort", " toutes les ordures", "sur ce fouillis", " de la poussière", "des toiles d'araignée". (Hugo, 2006 : 353)

En outre, la condition de vie solitaire de l'archidiacre dans ce lieu rappelle son enfance au collège de Torchi où il a vécu éloigné de ses parents, "cloîtré et comme muré dans ses livres...." (Hugo, 2006 : 199). La cellule toute haute et éloignée du monde des vivants revêt donc l'aspect d'un exil qui sépare Frollo du monde des hommes, qui tue en lui tout sentiment humain et qui en fait un être dur et inhumain. Ce n'est pas par hasard que l'auteur le compare souvent à une "statue"

"Eh bien! ma sœur, réjouissez-vous, dit le prêtre, glacial comme une statue de sépulcre..." (Hugo, 2006 : 454)

Ou encore:

" C'était un prêtre. On distinguait nettement son costume, et son visage appuyé sur ses deux mains. Du reste, il ne bougeait non plus qu'une statue. Son ceil fixe plongeait dans la place." (Hugo, 2006 : 323, 324)

Hugo nous dit à cet égard:

"Jusque-là il n'avait vécu que dans la science, il commençait à vivre dans la vie" (Hugo, 2006 : 198) 
C'est, tout de même, l'aveu final fait de la part du prêtre à la jeune fille et à travers lequel, il lui explique comment il était heureux avant de la voir, comment la science était toute sa vie et la chasteté le revêtait et à quel point sa vue l'a bouleversé et l'a mené à sa perte. A force d'être éloigné de la vie et enfermé dans sa cellule, l'archidiacre semble être ensorcelé par ce coup de foudre qui lui fit perdre son équilibre.

"Je t'attendais sous les porches, je t'épiais au coin des rues, je te guettais du haut de ma tour. Chaque soir, je rentrais en moi-même plus charmé, plus désespéré, plus ensorcelé, plus perdu!" (Hugo, 2006 : 443)

En fait le détour fatal des événements qui mène à la fin atroce de la jeune fille semble être le résultat de ce déséquilibre qui s'empare du prêtre dur et rude toujours enfermé dans la science et qui, influencé par la beauté de la Esméralda, cherche, tout d'un coup, à s'adonner aux plaisirs de la vie. Son "rire de démon" devant la scène d'exécution de la jeune fille décrit par l'auteur comme étant: "rire qu'on ne peut avoir que lorsqu'on n'est plus homme" symbolise l'état de dureté, d'inhumanité et de cruauté qui domine cet être cloîtré dès l'enfance loin du monde des vivants. Le très haut semble donc revêtir un aspect tout-à-fait négatif et au lieu de révéler la transcendance morale du prêtre, nous le trouvons revêtir un aspect tout-à-fait opposé soulignant sa décadence 
morale incarnée par l'aspect noir et sombre de sa cellule. C'est lui qui dit à la jeune fille:

" (...) je déchire mon nom; prêtre, je fais du missel un oreiller de luxure, je crache au visage de mon Dieu! tout cela pour toi, enchanteresse! pour être plus digne de ton enfer! et tu ne veux pas du damné!" (Hugo, 2006 : 641)

Il lui dit aussi:

" Eh bien, oui! assassin! dit-il, et je t'aurai. Tu ne veux pas de moi pour esclave, tu m'auras pour maître. Je t'aurai. (...) Il faut mourir, la belle, ou être à moi !(...) La tombe ou mon lit! » (Hugo, $2006: 643$ )

Le très haut, loin de refléter la transcendance morale est dans cette œuvre symbole de l'exclusion, de l'isolement, de l'éloignement du monde des vivants, de l'exil, de la fermeture et de tout ce que cela peut reproduire de durcissement, d'inhumanité, d'égoïsme et de narcissisme.

Avec toute leur solennité et leur grandeur, les tours de la cathédrale se transforment, pour Frollo, en tours de guet des déplacements de la jeune danseuse.

Ce très haut reproduit, de plus, un rapport de hiérarchie symbolique qui met l'archidiacre, en tant qu'homme de religion, au sommet de la pyramide hiérarchique sociale de son temps.

Pour le deuxième personnage fictif qui occupe le très haut, il s'agit de Quasimodo. En fait, on retrouve ce bossu partout dans la cathédrale. C'est lui qui donne de l'esprit à ce lieu solennel 
mais inerte et vide. Son va et vient ça et là est noté à la hâte mais l'auteur donne une autre dimension à son occupation du très haut de la cathédrale et l'appelle son sérail.

"Quasimodo (...) était monté jusque sur le sommet de la tour septentrionale" (Hugo, 2006: 557)

"(...) la grosse cloche était sa bien-aimée.

(Hugo, $2006: 207$ )

"Or, donner la grosse cloche en mariage à Quasimodo, c'était donner Juliette à Roméo." (Hugo, 2006 : 211)

"Quasimodo avait donc quinze cloches dans son sérail"

(Hugo, 2006 : 207)

Ce lieu assez haut éloigné toujours du monde des vivants semble être son sérail avec toutes les connotations que ce mot comporte. C'est là où Quasimodo se sent roi entre ses diverses femmes à savoir les quinze cloches des tours et dont la favorite est la cloche "Marie". C'est là où il crée un monde à sa guise, un monde qui le comprend et avec qui il peut enfin communiquer :

"Il est vrai que leur voix était la seule qu'il pût entendre encore." (Hugo, 2006 : 207)

C'est toujours là où on le trouve vivant, plein d'animations en contraste avec sa pesanteur partout ailleurs:

Parvenu dans la haute cage de la sonnerie, Quasimodo (...) allait et venait, il frappait des mains, il courait d'une corde à l'autre, il animait les six chanteurs de la voix et du geste, comme un chef d'orchestre qui éperonne des virtuoses intelligents." (Hugo, 2006 : 348) 
Et comme pour son père adoptif, les tours se transforment, pour Quasimodo, en lieu de guet du plan "bas" où se trouve la jeune bohémienne.

En passant devant la porte de la sonnerie qui était entr'ouverte, il vit une chose qui le frappa, il vit Quasimodo (...). Il était en proie à une contemplation si profonde qu'il ne prit pas garde au passage de son père adoptif. Son oil sauvage avait une expression singulière. C'était un regard charmé et doux. "Voilà qui est étrange! murmura Claude. Est-ce que c'est l'égyptienne qu'il regarde ainsi ? »(Hugo, $2006: 337)$

Toutefois, si l'occupation du trop haut de la cathédrale reflète toujours en quelque sorte l'isolement, l'éloignement et l'exil, dans le cas de Quasimodo, cette fermeture semble lui être imposée et revêt certains aspects de douleur, de souffrance et de solitude. Quasimodo n'a pas, par pur choix, abandonné la vie des humains. Par contre, le roman s'ouvre alors qu'il occupait "le bas" réservé à la collectivité, au peuple c'est-à-dire à tous les habitants de la capitale.

"Quasimodo, objet du tumulte, se tenait toujours sur la porte de la chapelle, debout, sombre et grave, se laissant admirer." (Hugo, 2006 : 75)

Pendant la fête des fous, il était toujours, sémiotiquement parlant, occupant "le bas", à la recherche d'une place parmi les hommes: 
"Cependant tous les mendiants, tous les laquais, tous les coupe-bourses, réunis aux écoliers, avaient été chercher (sic) (...) la tiare de carton et la simarre dérisoire du pape des fous. Quasimodo s'en laissa revêtir sans sourciller et avec une sorte de docilité orgueilleuse." (Hugo, 2006 : 75)

Mais la réaction des gens est toujours répulsive:

"Un écolier, Robin Poussepain, je crois, vint lui rire sous le nez" (Hugo, 2006 : 75)

Voir ausi:

"Quasimodo était sourd, mais il voyait clair, et la fureur publique n'était pas moins énergiquement peinte sur les visages que dans les paroles. D'ailleurs les coups de pierre expliquaient les éclats de rire." (Hugo, 2006 : 307)

L'éloignement, l'isolement, le très haut semblent donc lui être imposés à cause de sa difformité physique qui l'a expulsé, malgré lui, du monde des vivants pour lequel il garde un sentiment d'attrait instinctif reflété par son orientation vers le bas et sa recherche à occuper le plan "bas" d'où il est toujours expulsé.

"Après tout, il ne tournait qu'à regret sa face du côté des hommes." (Hugo, 2006: 307)

Cet attrait et cet instinct humain se reflète alors dans son comportement. Sa laideur physique ne l'empêche pas d'avoir un grand cœur sincère et reconnaissant et d'avoir une vertu cachée au fond de son âme souffrante. Il s'apitoie sur le sort de la jeune fille innocente et cherche à la sauver et reste reconnaissant et 
dévoué à l'égard de son père adoptif avant de se rendre compte de sa rigidité.

Le troisième personnage qui occupe le très haut est le roi Louis XI :

"Quasimodo (...) n'y voyait plus briller qu'une lumière, laquelle étoilait une vitre à l'étage le plus élevé d'un haut et sombre édifice, à côté de la porte Saint-Antoine. Cet édifice, c'était la Bastille. Cette étoile, c'était la chandelle de Louis XI." (Hugo, 2006: 581)

En fait ce dernier ne faisait que "de rares et courtes apparitions dans sa bonne ville de Paris". Une fois de plus, le très haut est mentionné pour souligner des valeurs négatives à savoir l'isolement du roi et son éloignement du peuple. Exilé dans le très haut et ne faisant que de rares apparitions, le roi est tout loin de ses ressortissants. Enfermé dans le faste et le luxe inouï, le roi semble être solitaire.

"Cette chambrette que le roi s'était réservée dans la fameuse prison d'état était encore assez vaste et occupait l'étage le plus élevé d'une tourelle engagée dans le donjon. C'était un réduit de forme ronde, tapissé de nattes en paille luisante, plafonné à poutres rehaussées de fleurs de lys d'étain doré avec les entrevous de couleur, lambrissé à riches boiseries semées de rosettes d'étain blanc et peintes de beau vert-gai, fait d'orpin et de florée fine." (Hugo, 2006: 581, 582) 
En fait, malgré tout ce faste, la chambrette du roi semble sombre et obscure et l'auteur en fait une sorte de cellule de prisonnier avec sa seule fenêtre où s'élèvent des barreaux et où le roi se trouve solitaire comme l'indique symboliquement la seule chaise existante:

"Il $n$ 'y avait qu'une fenêtre, une longue ogive treillissée de fil d'archal et de barreaux de fer, d'ailleurs obscurcie de belles vitres coloriées (...)"(Hugo, 2006: 582)

Lisons aussi:

"On ne trouvait dans cette chambre rien de ce qui meublait les appartements ordinaires, ni bancs, ni tréteaux, (...) On n'y voyait qu'une chaise pliante à bras, fort magnifique : le bois en était peint de roses sur fond rouge, le siège de cordouan vermeil, garni de longues franges de soie et piqué de mille clous d'or. La solitude de cette chaise faisait voir qu'une seule personne avait droit de s'asseoir dans la chambre." (Hugo, 2006: 582)

Isolé, enfermé et exilé par sa maladie ${ }^{3}$, par le faste et par l'éloignement, le roi semble être emprisonné dans une solitude atroce et presque incapable de communiquer avec son peuple. D'autant plus qu'il s'avère d'une dureté affreuse: son indifférence aux sanglots et aux supplications lamentables du prisonnier déjà incarcéré depuis quatorze ans et qui demande grâce en est la preuve. Hugo nous dit à cet égard:

"La voix lamentable qui était sortie de la cage avait glacé tous les assistants, maître Olivier lui-même. Le roi seul avait l'air de ne pas l'avoir entendue."(Hugo, 2006:592) 
En fait, le roi avait tout entendu et se rend compte de tout ce qui concerne ce prisonnier, Hugo nous confie cette réalité quelques lignes plus loin en disant:

"Le roi savait cela mieux que personne. Mais c'était une manie." (Hugo, 2006: 595)

Hugo use de sémantismes très évocateurs pour souligner l'état déplorable du prisonnier et l'impact de ses soupirs sur tous les assistants sauf le roi rigide, dur et inhumain tout-à-fait insoucieux des malheurs du damné.

\begin{tabular}{|c|c|}
\hline $\begin{array}{l}\text { Sémantismes évocateurs de l'état } \\
\text { du prisonnier }\end{array}$ & $\begin{array}{c}\text { Sémantismes évocateurs de } \\
\text { l'impact } \\
\text { des soupirs du prisonnier sur les } \\
\text { assistants }\end{array}$ \\
\hline 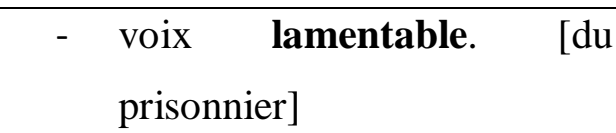 & $\begin{array}{llll}\text { - } & \text { avait glacé tous les } \\
\text { assistants } & & \end{array}$ \\
\hline $\begin{array}{l}\text { - on entendait sangloter le } \\
\text { prisonnier. }\end{array}$ & $\begin{array}{l}\text { - Cela était lugubre dans } \\
\text { l'ombre, et les visages se } \\
\text { regardaient en pâlissant. }\end{array}$ \\
\hline $\begin{array}{l}\text { - Et j'ai au pied une bien } \\
\text { lourde chaîne, et une grosse } \\
\text { boule de fer au bout, } \\
\text { beaucoup plus pesante qu'il } \\
\text { n'est de raison. Hé ! Sire ! } \\
\text { ayez pitié de moi! }\end{array}$ & $\begin{array}{l}\text { - Le roi remontait en silence à } \\
\text { son retrait, et son cortège le } \\
\text { suivait, terrifié des derniers } \\
\text { gémissements du condamné. }\end{array}$ \\
\hline
\end{tabular}

De tout ce qu'on a déjà cité, le très haut, loin de souligner la transcendance semble être dans ce roman symbole de 
l'isolement, de l'exil, de la solitude, de la souffrance, de la douleur et de l'éloignement. Plus on s'élève vers le haut, plus on s'éloigne des vivants, des valeurs humaines et plus on s'endurcit. Aussi, le roi et l'archidiacre occupant le tout haut apparaissent les plus durs et les plus inhumains. Plus on s'approche de la terre, plus on est doté d'émotions et de sentiments humains comme il est le cas de Quasimodo attiré intuitivement vers le monde des humains.

Pour le bas, comme on le voit dans le roman, il est occupé par le peuple, par la foule qui se précipite pour assister tantôt au Mystère, tantôt à la fête des fous, au pilori de Quasimodo ou au gibet de la jeune Esméralda. Il s'agit de ce peuple qui applaudit tout, qui approuve tout, qui est attiré indifféremment par le supplice et par l'acte charitable. C'est le même peuple qui assiste indifféremment au supplice de la jeune fille et qui, amoureux de toute prouesse, applaudit son sauvetage par Quasimodo. Hugo insiste à souligner la façon dont ils remplissent massivement les lieux: "amas de peuple", "encombré de peuple", "foison de peuple", "une foule immense". Leur occupation des terrains, des places et des lieux publics, autrement dit, $d u$ bas, semble significateur dans ce sens qu'il s'agit de la catégorie de la société la plus simple d'esprit qui est facilement guidée et aisément 
orientée. Il s'agit de cette catégorie qui occupe le bas de l'échelle sociale. Pour le trop bas, il faut noter qu'il est occupé par deux catégories dont l'une est encore plus basse que l'autre. La première est celle des truands, ensuite la seconde, celle des prisonniers.

A propos des truands, Hugo insiste à les situer à une échelle encore plus basse que celle du peuple. Pour s'y rendre dans leur lieu, Gringoire a dû suivre une "longue ruelle en pente non pavée boueuse et inclinée". Pour décrire ce lieu, "la cour des Miracles", Hugo use de sémantisme toujours à valeur négative comme "égout", "ruisseau de vice", "vaste place irrégulière et mal pavée", "l'horrible place", "la boue" etc. Et comme son nom l'indique*, il s'agit d'un lieu de miracles étant donné que l'aveugle y retrouvait la vue, le boiteux son second pied et le borgne y ouvrait largement de grands yeux. Pour amplifier le caractère hideux de ce lieu, Hugo compare ses habitants aux bêtes en les métaphorisant par l'emploi de substantifs et de prédicats tels : vagissement, griffe, moutonner et aboyer:

"On entendait des rires aigus, des vagissements d'enfants (...). (...) d'autres visages qui moutonnaient et aboyaient autour de lui (...). On l'entraîna. Ce fut à qui mettrait la griffe sur lui". (Hugo, 2006: 119,120) 
Quoique bas, ce monde était bien ordonné et avait ses propres lois et sa hiérarchie interne dont on parlera un peu plus loin ${ }^{4}$.

Quant à l'échelle le plus bas, nous trouvons Hugo, dans sa description des bâtiments du moyen âge, nous dire :

"Au moyen âge, quand un édifice était complet, il y en avait presque autant dans la terre que dehors. (...) Dans les cathédrales, c'était en quelque sorte une autre cathédrale souterraine, basse, obscure, mystérieuse, aveugle et тиеtte, sous la nef supérieure qui regorgeait de lumière et retentissait d'orgues et de cloches jour et nuit; quelquefois c'était un sépulcre. Dans les palais, dans les bastilles, c'était une prison, quelquefois aussi un sépulcre, quelquefois les deux ensemble." (Hugo, 2006: 432)

C'est dans un tel lieu que la jeune innocente était enfermée.

C'est dans un fond de cuve de ce genre, dans les oubliettes creusées par saint Louis, dans l'in-pace de la Tournelle, qu'on avait, de peur d'évasion sans doute, déposé la Esmeralda condamnée au gibet, avec le colossal Palais de Justice sur la tête. (Hugo, 2006: 433)

La formule ironique " de peur d'évasion" et l'expression figurative " avec le colossal Palais de Justice sur la tête" reflètent l'indignation et la révolte de V. Hugo contre ces procédures sévères et inhumaines surtout qu'elles contrastent avec la fragilité, la simplicité, la légèreté et l'innocence de la jeune fille de 17 ans *. Il continue à souligner l'aspect lugubre de ce lieu en usant un lexique relatif à la mort, à l'enterrement et à 
l'anéantissement tels : "les ténèbres", "ensevelie", "enfouie", "murée", "cette infortune", "ce cachot", "le néant" et "sépulcre". Il amplifie l'aspect lugubre de ce cachot en mentionnant sa noirceur jour et nuit :

"...(car minuit et midi avaient même couleur dans ce sépulcre)" (Hugo, 2006: 435)

En fait, la description du lieu et sa disposition sur l'axe vertical à un niveau encore plus bas que le bas servent surtout à refléter l'état d'exil, d'éloignement, d'emprisonnement sinon aussi cette situation de mort-vivant dont souffrent les damnés. Plus loin, vers la fin du roman, sur ce même plan trop bas, nous rencontrons un autre prisonnier, il s'agit là d'un personnage qui a réellement existé au Moyen Âge. Victor Hugo essaie de rappeler au lecteur les souffrances inouïes de ce damné alors oublié entre les hauts murs sans issue de la Bastille, il s'agit de l'évêque de Verdun. Là aussi, la description des lieux vient surtout incarner l'état de mort-vivant de la personne qui y réside. Les termes "tombe", "cage de fer", "cage outrageuse" etc en sont des exemples. L'espace est intimement lié à l'état moral de son occupant, il l'amplifie et le reproduit.

En ce qui concerne le "haut", ce grade semble être surtout occupé par le chevalier Phoebus, par sa fiancée et ses amies ainsi 
que par la famille de cette jeune fille. Ce lieu n'est ni trop haut comme le sommet de la cathédrale, ni trop bas comme les cachots, c'est un lieu à une échelle intermédiaire sur l'axe abstrait de la classification hiérarchique des personnages du roman. Du balcon de l'appartement de la famille de la jeune fiancée de Phoebus, les rayons de vision des jeunes filles, amies de Fleur de Lys, s'orientent vers le haut pour regarder l'archidiacre et vers le bas pour regarder la jeune bohémienne.

"Marraine, s'écria Bérangère dont les yeux sans cesse en mouvement s'étaient levés tout à coup vers le sommet des tours, de Notre-Dame, qu'est-ce que c'est que cet homme noir qui est là haut? Toutes les jeunes filles levèrent les yeux. (...)C'était un prêtre." [désignant l'archidiacre Claude Frollo] (Hugo, 2006: 323)

Lisons aussi:

"Le capitaine Phobus de Châteaupers (...) s'approcha à pas lents du balcon. "Tenez, lui dit Fleur-de-Lys (...) regardez cette petite qui danse là dans ce rond. Est-ce votre bohémienne?» (Hugo, 2006: 323)

La hauteur là dénote des catégories sociales plus privilégiées par rapport au peuple à savoir celles de la chevalerie et de la bourgeoisie auxquelles appartiennent les deux futurs époux. La place haute sans exagération qu'ils occupent fait partie du monde des humains avec toutes ses lois et ses valeurs. Ceux qui occupent ce lieu représentent de vrais types humains avec leurs tendances, leurs aspirations et leurs ambitions. C'est la seule catégorie présentée au 
sein d'une vie familiale et dont deux jeunes gens (Phoebus et Fleurde-Lys) planifient pour un futur engagement symbole de la continuité. C'est la seule catégorie qui survivra aux grandes mutations qui secoueront la France.

En fait, ce plan haut/bas peut être exploité une fois de plus pour mieux souligner une nouvelle dimension à savoir les rapports de force et de hiérarchie entre les diverses classes de la société de jadis. Cette fois, loin de s'intéresser à la localité qu'occupe chacun des personnages, nous allons alors étudier le rapport haut/ bas dans les scènes de confrontation des personnages appartenant à diverses classes sociales. Nous prenons quelques exemples pour mieux élucider l'idée.

Tout d'abord, dans les chapitres I et III du livre premier, intitulés La grand'salle et Monsieur le cardinal, Hugo dénote bien, sur l'axe de la verticalité, les places réservées aux personnalités imminentes et celles accordées à la population. Outre la chapelle réservée à Louis XI, il y a une "estrade de brocart d'or, adossée au mur, et dans laquelle était pratiquée une entrée particulière au moyen d'une fenêtre du couloir de la chambre dorée, (...) pour les envoyés flamands et les autres gros personnages conviés à la représentation du mystère"( 20. 
Par contre, le reste de la population occupe le parterre, on dit à cet égard qu'il y avait "un redoublement de tumulte et de curiosité au parterre" (Hugo, 2006: 52)

L'écolier, lui aussi, par l'enseignement qui lui est accordé à une époque où l'enseignement était réservé à l'élite privilégiée, occupe une place élevée par rapport aux autres. Il se trouve dans un chapiteau au haut d'une colonne.

" Jehan (...) on le distinguait encore au haut de son pilier." (Hugo, 2006: 71)

D'autre part, Seuls l'archidiacre, le notaire, l'officier se trouvent en selle sur le dos d'une mule ou d'un cheval ${ }^{5}$ par rapport au reste de la population qui se déplace à pied. Notons bien que la jeune Esméralda fut ravie d'être sauvée par le chevalier et de se trouver sur selle devant lui. C'est à partir de ce moment qu'elle se trouve éblouie par le capitaine. Son rapprochement de Phoebus lui permet donc une certaine ascension sociale symbolisée par ce geste d'élévation de la jeune fille auprès de lui d'où son idée de se marier au capitaine. Ce qu'il prend pour folie et ce pourquoi, elle se trouve obligée de s'excuser pour y avoir pensé :

"Eh bien, non! ne nous marions pas, cela t'ennuie. Et puis, qu'est-ce que je suis, moi? une misérable fille du ruisseau, tandis que toi, mon Phœbus, tu es gentilhomme. 
Belle chose vraiment! une danseuse épouser un officier! j'étais folle. Non, Phobus, non, je serai ta maîtresse, ton amusement, ton plaisir (...)" (Hugo, 2006: 404)

En fait, les rayons du regard de la jeune fille s'orientent toujours vers le haut pour voir son Phoebus. Ce qui reflète, en quelque sorte, le fossé social qui les sépare. De même, le plan bas (la rue) qu'occupe toujours la jeune bohémienne par rapport à Fleur de Lys et à ses amies qui se réservent souvent un plan plus haut (le balcon de leur appartement) reste lui aussi révélateur de sens. La posture debout, les yeux baissés de la Esméralda sur le seuil de l'appartement de Fleurs-de-Lys témoignent une fois de plus de cette distance sociale qui les sépare. Même, entre bandits, Hugo insiste à dessiner ce rapport hiérarchique en plaçant le roi des truands sur un tonneau pour lui réserver une place plus haute par rapport aux membres du groupe *.

"Un tonneau était près du feu, et un mendiant sur le tonneau. C'était le roi sur son trône." (Hugo, 2006: 122)

C'est ainsi que Hugo laisse insinuer au lecteur le rapport d'hiérarchie dominant les relations sociales de jadis.

En somme, nous pouvons dire que vu le caractère perceptible des lieux, ils ont été exploités par l'auteur pour reproduire des valeurs plus ou moins abstraites. Les axes haut/ bas ont constitué des espaces imaginaires d'appartenance ou non 
au monde des humains et aux valeurs humaines communes. Ils reflètent aussi la hiérarchie sociale de l'époque médiévale en situant chacun des personnages à un niveau correspondant à la catégorie à laquelle il appartient.

\subsection{Etude des relations spatiales binaires relevant du} concept : (ouvert/fermé ou extérieur/intérieur)

Revenons à une autre relation binaire, celle dérivée du concept ouvert/fermé ou extérieur/intérieur. Cette relation semble être elle aussi bien exploitée par l'auteur. Avec la grande diversité des personnages de l'œuvre appartenant aux diverses classes sociales, il a fallu trouver un moyen permettant leur rencontre et leur interaction. Dans ce roman, on trouve à la fois l'archidiacre, le chevalier, l'écolier, le poète, la bohémienne et le bossu aussi bien que des juges, des sergents, des chanceliers et son éminence le roi Louis XI. Des foules de Parisiens sont présents mais aussi des truands et des troupes du roi. Dans le carrefour de la vie, étant donné que ces gens appartiennent à des mondes différents et à des modes de vie tout-à-fait opposés, ils ne se réunissent que rarement dans des lieux fermés. La route, les places publiques ou plutôt l'extérieur et les lieux ouverts semblent représenter ce carrefour de la vie où tout s'entremêle. Et comme le dit Bakhtine: 
Dans les romans, les rencontres se font, habituellement, " en route ", lieu de choix et des contacts fortuits ». Sur la grande route se croisent au même point d'intersection spatio-temporel les voies d'une quantité de personnes appartenant à toutes les classes, situations, religions, nationalités et âges. [...] (1978: 384)

Ces lieux abondent dans le roman permettant alors la rencontre et le croisement de ces mondes divers pour une meilleure progression de l'intrigue romanesque. La place de Grève, la place du Palais, les avenues du Palais de Justice et la Cour des Miracles en sont des exemples.

Cependant, certains personnages du roman semblent être créés pour vivre dans telle ou telle ambiance, le changement de lieu menace leur existence, leur paix intérieure ou même la paix des autres. La jeune vagabonde, par exemple, et comme l'adjectif vagabond l'indique, semble être créée pour errer ça et là; l'enfermer ou l'emprisonner, c'est en quelque sorte la faire tuer. Hugo a bien démontré cette réalité. Son Esméralda est créée pour vivre à sa guise sans chaînes ni clôtures. Nous la trouvons animer la scène, éblouir et émerveiller son entourage chaque fois qu'elle occupe un lieu ouvert avant d'être accablée par sa fatalité destructrice.

" (...) la Esmeralda! la Esmeralda dans la place!»" 
Ce mot produisit un effet magique. Tout ce qui restait dans la salle se précipita aux fenêtres, grimpant aux murailles pour voir, et répétant : la Esmeralda! la Esmeralda!

(Hugo, 2006: 79)

Ou encore:

"Autour d'elle tous les regards étaient fixes, toutes les bouches ouvertes; et en effet, tandis qu'elle dansait ainsi, au bourdonnement du tambour de basque que ses deux bras ronds et purs élevaient au-dessus de sa tête, mince, frêle et vive comme une guêpe (...)" (Hugo, 2006: 91)

Par contre, elle s'éclipse et perd sa gaîté chaque fois qu'elle se trouve clôturée par des murs ou des cloisons. C'est dans la chambre à Sainte-Marthe que commencent ses chagrins. C'est entre ces quatre murs qu'elle rate une nuit d'amour et fut accusée du meurtre de son bien-aimé:

Quand elle reprit ses sens, elle était entourée de soldats du guet, on emportait le capitaine baigné dans son sang, (...) et elle entendait dire autour d'elle : "C'est une sorcière qui a poignardé un capitaine.» (Hugo, 2006: 405)

C'est aussi entre les murs du palais de la justice et surtout dans la chambre de la question qu'elle souffre l'injustice, la cruauté et la terreur:

Cependant la Esmeralda restait debout. Ce lit de cuir, où s'étaient tordus tant de misérables, l'épouvantait. La terreur lui glaçait la moelle des os. Elle était là, effarée et stupide. (Hugo, 2006: 422) 
C'est entre les murs du cachot du palais de la justice qu'elle frémit de chagrin et de peur:

Elle était là, perdue dans les ténèbres, ensevelie, enfouie, murée. Qui l'eût pu voir en cet état, après l'avoir vue rire et danser au soleil, eût frémi. Froide comme la nuit, froide comme la mort (...). (Hugo, 2006: 433)

Même la fermeture dans Notre-Dame semble l'étouffer. Quoique la cathédrale représente pour la jeune fille la valeur de la protection et constitue, pour elle, un refuge contre la mort. Sa présence dans ce lieu fermé l'ennuie et la cathédrale se transforme vite, pour elle, en prison qui l'éloigne de la vie :

La malheureuse! elle était plus hors du monde que Quasimodo! Hugo, 2006: 505)

En fait, le cheminement spatial de la bohémienne se fait dans un cercle vicieux. Elle suit un trajet circulaire, allant d'un lieu ouvert (la place de Grève) vers des lieux fermés pour se trouver toujours sur ce même lieu ouvert (la place de Grève). Ce lieu sinistre, semble la destination fatale et inévitable de la jeune fille. Elle s'en va ça et là pour se trouver toujours face à face au pilori de la Grève où sera mis fin à ses jours. Le destin est comme un aimant qui l'attire involontairement vers sa fatalité. Son cheminement alternatif entre lieux ouvert et fermé la mène incontestablement à la mort. Ce lieu de supplice constitue donc une station essentielle dans son cheminement spatial. Elle s'y 
trouve conduit inconsciemment. Nous soulignons, dans le schéma suivant, les déplacements alternatifs ouvert/fermé de la jeune fille qui ponctuent son passage de l'état initial 1 où elle était joyeuse vers l'état final 2 où elle fut menée fatalement à la mort. Ce cheminement souligne l'ascendance graduelle de ses malheurs: allant de ses danses et ses chants gais sur la place de la Grève jusqu'à sa pendaison finale en passant par son accusation, son jugement tortueux, son emprisonnement, son gibet manqué et sa fermeture dans la cathédrale auprès de son grand ennemi, le prêtre, avant d'être livrée à la récluse qui en cherchait vengeance.

Lieu ouvert

(La place de grève)

(Danse et gaîté)

Lieu fermé

(La chambre de Sainte-Marthe, le palais de justice, la chambre de la question, le cachot)

Lieu ouvert

(La place de grève)

(Gibet manqué)

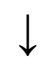

$-1 \leqslant 1$. 
Lieu fermé

(La cathédrale, la cellule de la recluse)

Lieu ouvert

(La place de grève)

(Exécution)

Toutefois, comme ce lieu est à la fois lieu de fête et de supplice, lieu de joie et de mort, son caractère double se manifeste sur le sort de la jeune fille*. Ayant paru pour la première fois enchantée et rayonnante sur la place de Grève, la jeune tsigane y revient vers la fin après tant de déception et d'injustice humaine pour subir son sort fatal et être exécutée. L'ombre de son corps vacillant par la danse et reflété par le feu de joie sur la Maison aux Pilliers et sur le gibet au début du roman dans le chapitre III du livre deuxième prédit sa fin atroce, tordue et dont le corps se balance au bout de la corde dans le chapitre II du neuvième livre. C'est là où on la voit pour la première fois avec tout son charme, sa beauté et son animation. C'est là où son destin la conduit innocente au gibet manqué; et c'est toujours là où le sort la fait revenir à la fin pour être exécutée.

Par contre, Quasimodo, le jeune bossu, semble être créé pour vivre à l'abri, loin des gens pour éviter leur moquerie et leur 
méchanceté. Chaque fois qu'il se trouve parmi eux, il est le sujet de leur raillerie et de leur moquerie ${ }^{6}$. Aussi, la cathédrale, ce lieu fermé, lui offre la protection et se transforme pour lui en logis et abri. Elle devient sa famille et lui offre ses cloches pour favorites et ses statues pour amis fidèles. La cathédrale devient donc sa société et son seul univers. C'est pourquoi, il la défend avec acharnement contre les truands. Dans un cheminement toujours circulaire, l'histoire de ce modeste être difforme, enfant trouvé, commence alors qu'il est étendu horizontalement sur le parvis de l'église dans l'attente d'un parrain charitable et sa vie se termine toujours étendu horizontalement serrant le corps mort de sa bienaimée. Cette posture horizontale, la moins détectée dans le roman, souligne l'état d'inertie, de lassitude, de faiblesse et de détente que lui a imposé sa déformation physique.

Pour l'archidiacre dont l'habitude est la fermeture et la solitude, nous pouvons considérer toute interaction de sa part avec le monde extérieur et toute sortie de son exil même quand elle a lieu dans un autre lieu fermé comme étant un passage du fermé à l'ouvert, de la solitude à l'interaction, de la cellule vers le monde extérieur. Mais pour plus d'exactitude, nous avons préféré passer à une autre relation spatiale binaire, celle inspirée du concept proche/lointain. 


\subsection{Etude de la relation spatiale binaire (proche/ lointain(e)):}

L'archidiacre chez Hugo semble être le démon du roman dont le rapprochement envenime la vie des autres et surtout celle de la jeune tsigane. Se rapprochant de Phoebus, il le poignarde; se rapprochant de la jeune fille, il la rend malheureuse et la mène à la mort. Croisant la voie de la recluse, il la mène au chagrin et à la mort. Ses soins accordés au petit bossu en fait un esclave sinon un valet ${ }^{7}$. Même ses soins à l'égard de son frère "Jehan" le gâte. Il s'agit d'un personnage qui sème le malheur sur la voie des autres. Pour mieux expliquer l'idée, nous prenons l'exemple le plus évident, ses rapprochements de la jeune fille et ses diverses tentatives pour braver sa voie. Le tableau suivant notera chaque rapprochement spatial entre l'archidiacre et la jeune tsigane tout en soulignant la réaction négative de la jeune fille:

\begin{tabular}{|c|c|}
\hline $\begin{array}{c}\text { Rapprochement spatial entre le } \\
\text { prêtre } \\
\text { et la Esméralda. }\end{array}$ & Réaction de la jeune fille \\
\hline $\begin{array}{l}\text { "Il y a de la sorcellerie là-dessous », dit une } \\
\text { voix sinistre dans la foule. C'était celle de } \\
\text { l'homme chauve qui ne quittait pas la } \\
\text { bohémienne des yeux. }\end{array}$ & $\begin{array}{l}\text { Elle tressaillit, se détourna (...) (Hugo, } \\
\text { 2006: 94) }\end{array}$ \\
\hline $\begin{array}{l}\text { "Cet homme, ce téméraire, c'était le } \\
\text { personnage au front chauve qui, (...)." }\end{array}$ & $\begin{array}{l}\text { (...) avait glacé la pauvre fille de ses } \\
\text { paroles de menace et de haine. (Hugo, } \\
\text { 2006: 100) }\end{array}$ \\
\hline "C'était la figure et la main du prêtre." & $\begin{array}{l}\text { "La jeune fille resta immobile, glacée, } \\
\text { muette sous l'épouvantable apparition } \\
\text { (..)."(Hugo, 2006: 405) }\end{array}$ \\
\hline «Qui êtes-vous ? [demande la jeune fille & Le mot, l'accent, le son de voix, la firent \\
\hline
\end{tabular}




\begin{tabular}{|c|c|}
\hline $\begin{array}{l}\text { au prêtre dont la face est cachée sous son } \\
\text { capuchon] } \\
\text { - Un prêtre. » }\end{array}$ & tressaillir. (Hugo, 2006: 436) \\
\hline $\begin{array}{l}\text { "En parlant ainsi, le prêtre lui prit le bras." } \\
\text { [La face du prêtre est toujours cachée.] }\end{array}$ & $\begin{array}{l}\text { La malheureuse était gelée jusque dans les } \\
\text { entrailles, cependant cette main lui fit une } \\
\text { impression de froid. } \\
\text { "Oh ! murmura-t-elle, c'est la main glacée } \\
\text { de la mort.(..)" (Hugo, 2006: 437) }\end{array}$ \\
\hline $\begin{array}{l}\text { "Le prêtre releva son capuchon. Elle } \\
\text { regarda. C'était ce visage sinistre qui la } \\
\text { poursuivait depuis si longtemps, cette tête de } \\
\text { démon qui }(\ldots) \text { "... }\end{array}$ & $\begin{array}{l}\text { «Hah ! cria-t-elle, les mains sur ses yeux et } \\
\text { avec un tremblement convulsif, c'est le } \\
\text { prêtre! » } \\
\text { Puis elle laissa tomber ses bras } \\
\text { découragés, et resta assise, la tête baissée, } \\
\text { l'œil fixé à terre, muette, et continuant de } \\
\text { trembler. (Hugo, 2006: 438) }\end{array}$ \\
\hline $\begin{array}{l}\text { La nuit était très noire. Cependant elle vit à } \\
\text { sa lucarne une figure qui la regardait. Il y } \\
\text { avait une lampe qui éclairait cette } \\
\text { apparition. Au moment où elle se vit } \\
\text { aperçue de la Esmeralda, cette figure souffla } \\
\text { la lampe. Néanmoins la jeune fille avait eu le } \\
\text { temps de l'entrevoir. [Il s'agit du prêtre] }\end{array}$ & $\begin{array}{l}\text { Ses paupières se refermèrent de terreur. } \\
\text { «Oh ! dit-elle d'une voix éteinte, le prêtre } \\
! » \\
\text { Tout son malheur passé lui revint comme } \\
\text { dans un éclair. Elle retomba sur son lit, } \\
\text { glacée. } \\
\text { Un moment après, elle sentit le long de son } \\
\text { corps un } \\
\text { contact qui la fit tellement frémir qu'elle } \\
\text { se dressa réveillée et furieuse sur son } \\
\text { séant. (Hugo, 2006: } 520 \text { ) }\end{array}$ \\
\hline $\begin{array}{l}\text {-Soyez tranquille, répondit Gringoire. C'est } \\
\text { un de mes amis. » [dit Gringoire désignant } \\
\text { le prêtre dont la face est cachée sous le } \\
\text { capuchon et sans savoir la nature de la } \\
\text { relation répulsive liant la jeune fille et le } \\
\text { prêtre.] }\end{array}$ & $\begin{array}{l}\text { La peur étourdissait la jeune fille. (Hugo, } \\
\text { 2006: 631) }\end{array}$ \\
\hline $\begin{array}{l}\text { "(..) elle était seule sur le port avec } \\
\text { l'inconnu." }\end{array}$ & $\begin{array}{l}\text { La pauvre égyptienne frissonna de se voir } \\
\text { seule avec cet homme. Elle voulut parler, } \\
\text { crier, appeler Gringoire, sa langue était } \\
\text { inerte dans sa bouche, et aucun son ne } \\
\text { sortit de ses lèvres. (Hugo, 2006: 637) }\end{array}$ \\
\hline $\begin{array}{l}\text { "Tout à coup elle sentit la main de l'inconnu } \\
\text { sur la sienne." }\end{array}$ & $\begin{array}{l}\text { C'était une main froide et forte. Ses dents } \\
\text { claquèrent, elle devint plus pâle que le } \\
\text { rayon de la lune qui l'éclairait. (Hugo, } \\
\text { 2006: 637) }\end{array}$ \\
\hline
\end{tabular}




\begin{tabular}{|l|l|}
\hline $\begin{array}{l}\text { "L'homme s'arrêta, se tourna vers elle, et } \\
\text { leva sa carapoue." }\end{array}$ & $\begin{array}{l}\text { «Oh ! bégaya-t-elle pétrifiée, je savais } \\
\text { bien que c'était encore lui ! » (Hugo, 2006: } \\
638)\end{array}$ \\
\hline
\end{tabular}

Comme on le voit, les prédicats : tressaillir, glacer, trembler, frémir, étourdir, frissonner, claquer, bégayer; les adjectifs: immobile, glacée, muette, gelée, furieuse, inerte, pâle, pétrifiée et les substantifs: menace, haine, froid, mort, tremblement, malheur, tous concourent à décrire l'état de tension, de malaise, de souffrance et de terreur où se trouve la jeune fille chaque fois que ce démon se rapproche d'elle. Cette réaction de tressaillement et de soubresaut devient un fait intuitif. N'oublions pas que dans les cas 4, 5, 8, 9 et 10 du tableau précédent, la jeune tsigane avait éprouvé ce sentiment de terreur et de frémissement avant même de se rendre compte qu'elle était en compagnie de cet homme horrible qui la poursuivit et qui sema sa vie de malheurs continuels.

Par contre, le rapport proche/ lointain entre la bohémienne et Phoebus semble revêtir un aspect tout-à fait différent. Son rapprochement semble la combler de joie et c'est sa disparition qui excite sa tristesse. Le tableau suivant démontre l'effet réjouissant de son rapprochement: 


\begin{tabular}{|c|c|}
\hline $\begin{array}{c}\text { Rapprochement spatial entre le } \\
\text { capitaine } \\
\text { et la Esméralda. }\end{array}$ & Réaction de la jeune fille \\
\hline $\begin{array}{l}\text { "Il arracha la bohémienne des bras de } \\
\text { Quasimodo stupéfait, la mit en travers sur sa } \\
\text { selle (...)." } 107\end{array}$ & $\begin{array}{l}\text { La bohémienne }(\ldots) \text { le regarda fixement } \\
\text { quelques secondes, comme ravie de sa } \\
\text { bonne mine }(\ldots) .(\text { Hugo, 2006: 108) }\end{array}$ \\
\hline $\begin{array}{l}\text { "Quand il revint à lui, Phoebus et la } \\
\text { Esmeralda étaient seuls }(\ldots) "\end{array}$ & $\begin{array}{l}\text { La jeune fille était rouge, interdite, } \\
\text { palpitante. (Hugo, 2006: 396) }\end{array}$ \\
\hline $\begin{array}{l}\text { "Phobbus vint se rasseoir près d'elle, mais } \\
\text { beaucoup plus près qu'auparavant." }\end{array}$ & $\begin{array}{l}\text { "L'égyptienne lui donna quelques petits } \\
\text { coups de sa jolie main sur la bouche avec } \\
\text { un enfantillage plein de folie, de grâce et } \\
\text { de gaieté." (Hugo, 2006: 399) }\end{array}$ \\
\hline $\begin{array}{l}\text { À ce balcon, là bas, à l'angle de la place, elle } \\
\text { venait de l'apercevoir, lui, son ami, son } \\
\text { seigneur, Phoebus, l'autre apparition de sa } \\
\text { vie! }\end{array}$ & $\begin{array}{l}\text { Tout à coup, tandis que l'homme jaune lui } \\
\text { liait les } \\
\text { coudes, elle poussa un cri terrible, un cri } \\
\text { de joie. (Hugo, 2006: } 474 \text { ) }\end{array}$ \\
\hline $\begin{array}{l}\text { " Oh ! le voilà qui descend de cheval ! - Il va } \\
\text { entrer dans cette maison ! - Phobbus ! - Il } \\
\text { ne m'entend pas ! - Phœebus !" [dit la jeune } \\
\text { tsigane] }\end{array}$ & $\begin{array}{l}\text { Elle poussa un cri de joie. «Oh ! va ! allez } \\
! \text { cours ! vite ! ce capitaine ! ce capitaine ! } \\
\text { amenez-le-moi ! je t'aimerai ! » (Hugo, } \\
\text { 2006: 508) }\end{array}$ \\
\hline
\end{tabular}

En fait, la joie s'empare de la jeune fille non seulement à son rapprochement mais aussi à sa vue, à l'écoute de son nom ou de sa voix.

Donc les deux apparitions de sa vie provoquent chez la jeune tsigane deux effets contrastifs. L'un excite sa terreur et son effroi tandis que l'autre excite sa joie et sa tendresse. L'analyse du rapport spatial proche/ lointain et de sa relation avec l'état d'âme de l'héroïne du roman le prouve. Il en est de même pour Quasimodo dont le rapprochement de la Esméralda excite toujours la joie. Cependant, le rapport proche/lointain entre d'autres personnages du roman ne donne pas toujours un effet 
stable d'effroi ou de joie qui se prolonge tout le long du roman comme il est le cas pour ces deux pôles magnétiques qui attirent et relâchent la jeune fille. On a pu noter des rapports transformationnels comme le rapport proche/lointain entre la Esméralda et la récluse. Alors que les rapprochements de la récluse excitaient à travers tout le roman le sursaut et le frémissement de la jeune bohémienne, nous trouvons que vers la fin du roman, et une fois découvrant la relation de filiation qui les lie, le rapprochement de la récluse rassure la jeune fille et la défend. De même, le rapprochement entre le bossu et le prêtre qui représente tout le long du roman de la part de Quasimodo un rapport de respect, de protection, de soumission et de reconnaissance se transforme vers la fin en rapport de vengeance qui le mène à le pousser du haut de la cathédrale.

Comme on le voit donc, les relations spatiales binaires, loin de se limiter à préciser les localités réservées à chacun des personnages, sont bien exploitées pour mieux élucider la fiction, pour bien souligner certains caractères des personnages aussi bien que leur cheminement transformatif.

Outre ces rapports spatiaux binaires, l'espace, comme on l'a déjà mentionné au début du travail et comme le démontre les 
travaux de Marie Laure $\operatorname{Ryan}^{8}$, peut aussi revêtir une valeur métaphorique.

\section{Etude métaphorique de l'espace :}

\subsection{L'analogie:}

Dans ce cas, le nom d'un lieu est intimement lié aux faits qui s'y déroulent. La cour des miracles en est un exemple ${ }^{9}$. Le trou aux rats représente lui-aussi un nom métaphorique reflétant les conditions de vie misérable que s'impose celle qui vit volontairement dans ce lieu sépulcral. Notons bien que le vrai nom de ce lieu est Tu, Ora qui signifie "Toi, prie" en latin, nom relatif à la destination pieuse que la défunte propriétaire voulait accorder à cette cavité. Mais, ce nom se transforme vite par les Parisiens en "Trou aux Rats" nom reproducteur de l'aspect sombre et modeste de cette cellule et lié à la condition de vie de celle qui opte volontairement de l'occuper :

Ce qui fait que le peuple, dont le bon sens ne voit pas tant de finesse dans les choses (...) avait donné à cette cavité noire, sombre et humide, le nom de Trou aux Rats. (Hugo, 2006: 273)

Notons bien que le choix du nom de ce logis est bien justifié. Il s'agit d'un lieu creusé dans la muraille sans porte et dont la petite lucarne est fermée de barreaux laissant à peine faire entrer l'air suffisant à celle qui y vit. Sa propriétaire et première 
habitante y dormait dans la cendre, sans même une pierre sous la tête. Elle était vêtue d'un sac noir et ne vivait que de ce que les passants déposaient sur sa lucarne. Le lieu était réservé à celles qui voulaient s'enterrer vives dans la douleur ou comme pénitence d'une faute commise ${ }^{10}$. Comme un rat, celle qui y habitait, ne faisait rien. Elle vivait en parasite au dépend des autres menant une vie misérable et modeste sans but, ni ambitions et n'attendant que la fin de ses jours.

Le nom de la rue "Folle Peine" est chargé lui-aussi d'un aspect métaphorique et analogique dans ce sens que cette rue était le témoin de la folle peine qui s'empare de la Chantefleurie après la disparition de sa fille unique. Pour décrire l'amour de la Chantefleurie envers sa fille, Hugo nous dit:

Sa mère en devenait de plus en plus folle tous les jours. Elle la caressait, la baisait, la chatouillait, la lavait, l'attifait (...) (Hugo, 2006: 284)

Et pour décrire sa détresse après sa disparition, il raconte l'angoisse, la folie et la peine qui s'emparent de cette mère et lui font grisailler les cheveux du jour au lendemain.

\subsection{L'opposition:}

Outre ce rapport d'analogie entre le lieu et les événements qui s'y déroulent, Hugo fait revêtir certains autres lieux d'un rapport d'opposition. Cette relation relève toujours du rapport 
métaphorique et sert non seulement à attirer l'attention du lecteur mais aussi à critiquer et à souligner certaines pratiques horribles ou à mettre en relief certaines grandes transformations romanesques dont l'espace est un acteur significatif de poids ou tout simplement un témoin muet.

C'est dans le palais de justice par exemple (supposé être le lieu de l'équité par excellence) que la Esméralda subit les plus grandes injustices. Elle fut accusée du meurtre d'un vivant et subit la torture pour avouer un crime dont elle est totalement innocente. C'est dans une cavité souterraine de ce lieu grandiose que la jeune innocente de 17 ans fut enfermée avec tout le palais de justice sur la tête de peur de sa fuite ${ }^{11}$. C'est toujours dans ce palais de justice que Quasimodo fut condamné innocent pour la seule faute d'être sourd et incapable de se défendre.

La place de Grève est elle aussi à la fois lieu de grandes fêtes, de grands loisirs, de cris de joie et de jubilation et lieu du supplice, de la mort, des cris de rage et d'horreur. Cette place semble représenter en quelque sorte le caractère et la condition de l'héroïne du roman ${ }^{12}$. De même, la chambre louée chez la Falourdel se transforme d'un lieu témoin d'une scène d'amour en lieu témoin d'une tentative d'assassinat qui bouleverse la vie de la jeune fille de fond en comble. 
Comme on le voit, la nomination des lieux ne revient pas au hasard, elle les dote d'une certaine valeur métaphorique d'analogie ou d'opposition significative qui charge ainsi l'espace d'une valeur sémantico-sémiotique révélatrice de sens.

\section{Conclusion}

De tout ce qui précède, on peut conclure que l'espace dans Notre Dame de Paris, loin de se limiter à la configuration esthétique et physique des lieux et des monuments de la capitale parisienne menacés de disparition, revêt d'autres valeurs significatives et fictives importantes. Les divers marqueurs spatiaux développés dépassent le cadre spatial et renvoient à des significations beaucoup plus abstraites. Ils renforcent la fiction et la reproduisent grâce à leurs connotations métaphoriques et à leurs relations binaires porteuses de sens. Ils soulignent les divers rapports de force et de hiérarchie entre les diverses classes de la société de jadis, élucident le caractère, la condition et les souffrances de certains personnages, rythment certains déplacements et transformations des héros.

En un mot, par ses connotations sémantiques et sémiotiques, l'espace semble être l'écho des événements et des péripéties romanesques qu'il renferme. Il est aussi témoin de la cruauté, de l'ingratitude et de l'injustice humaine. Comme 
l'espace, le temps semble être un des éléments évocateurs bien exploités par l'auteur et qui ouvre une nouvelle perspective d'analyse riche en connotations multiples.

\section{Bibliographie:}

Les ouvrages cités dans la bibliographie sont tous consultés même ceux qui ne figurent pas dans les notes de la recherche.

\section{- Le Corpus :}

Hugo (Victor), Notre-Dame de Paris 1482, Ebooks libres et gratuits $82 \mathrm{H}$ http://fr.groups.yahoo.com/group/ebooksgratuits, juin 2006.

\section{- Ouvrages de référence :}

- Bakhtine ( Mickhail), Esthétique et théorie du roman, Paris, Gallimard, 1978.

- Baylon (Christian) et Mignot (X.), Sémantique du langage initiation-, Paris, Nathan, 1995.

- Genette (Gérard), Figure II, Paris, Editions du Seuil, 1969.

- Issacharoff (Michael), Le spectacle du discours, Paris, José Corti, 1985.

- Kerbrat-Orecchioni (Catherine),

- Les interactions verbales II, Paris, Armand Colin, 1992.

- Les interactions verbales III, Paris, Armand Colin, 1994.

- Lotman, (Y.), La Structure du texte artistique, Paris, Ga

- Articles parus dans des collections, des colloques et des périodiques:

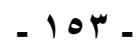


- Anonyme, "Sémiotique et sémantique de l'espace dans l'œuvre de Kateb Yacine, Nedjma", article extrait d'une thèse en " cours de fabrication » in Revue texto, pp. 1- 20. Consulté en janvier 2015.

- URI: http:www.revue-texto.net nedjma_navarette

- Chol (Isabelle), "Espace d'observation, espace d'écriture : questions de théorie et de méthode" in Revue d'études françaises, $\mathrm{n}^{\circ}$ 3, 2013, pp 2-14. Consulté en novembre 2015.

- URI: http://id.erudit.org/iderudit/1017370ar

- Craig (Robin), "Imaginaire des lieux dans le roman Malvina" in Revue d'études françaises, $\mathrm{n}^{\circ} 3,2013$, pp. 2 - 15. Consulté en décembre 2015.

- URI: http://id.erudit.org/iderudit/1017366ar

- Lamizet (Bernard), "La sémiotique de l'évènement" in Hal. archives ouvertes, pp.1-7. Consulté en Octobre 2015.

- URI: https://halshs.archives-ouvertes.fr/halshs-00604452

- Mitu (Mihaela), "L'espace mode de vision et représentation sémiotique dans l'œuvre de Michel Tournier", Les travaux de Universitatea din Pitesti, pp. 577- 583. Consulté en Octobre 2015.

- URL: http://www.upm.ro conferinte Lucrari Mitu>

- Ryan, M.-L. 2009. « Space ». Handbook of Narratology. P. Hühn, J. Pier, W. Schmid et J. Schönert (dirs.). Berlin ; New York : Walter de Gruyter, 420-433. (en ligne: http://www.lhn.unihamburg.de/article/space. Consulté le 4 décembre 2015). 
- Song (ki-Jeong), La Sémiotique de l'espace dans l'œuvre de Le Clézio. Le cas de La Quarantaine, in the 10th World Congress of the International Association for Semiotic Studies (IASS/AIS) Universidade da Coruña (España / Spain), 2012, pp. 371-382. Consulté en mars 2015.

- URI: http:ruc.udc.es bitstream CC-130_art_37

- Ziethen (Antje), «La littérature et l'espace » in Revue d'études françaises, $\mathrm{n}^{\circ}$ 3, 2013, pp. 2 -29. Consulté en janvier 2015.

- URI: http://id.erudit.org/iderudit/1017363ar 
1 Si les travaux de Michael Issacharoff s'intéressent surtout à l'analyse de l'espace théâtral, il faut bien souligner qu'on a bien profité des comparaisons qu'il a tenu à établir entre l'espace dramatique et l'espace romanesque aussi bien que de ses idées sur les rapports ouvert/clos, vertical/horizontal etc.

2 Nous proposons cette traduction de la citation : "Lorsque la notion d'espace réfère à un modèle formel, elle est prise dans un sens métaphorique puisqu'il ne s'agit pas d'un système de dimensions qui détermine la position physique, mais plutôt d'un réseau de relations analogiques ou oppositionnelles perçues par l'esprit. C'est la perspective synchronique qui est nécessaire pour la perception de ces (designs) conceptions et c'est la tendance à associer ce qui est synchrone à ce qui est spatial qui les catégorise comme des phénomènes spatiaux."

3 " (...) le vieux roi malade et voûté, qui toussait en marchant. À chaque guichet, toutes les têtes étaient obligées de se baisser excepté celle du vieillard plié par l'âge. " Hum ! disait-il entre ses gencives, car il n'avait plus de dents(...)"

- Hugo (V.), Op.cit., p. 590.

4 Voir p. $12(*)$

5 Voir Hugo (V.), Op.cit., pp. 105, 308.

6 Voir p. 6.

7 Voir Hugo (V.), Op.cit, p. 211.

8 Voir la page 2.

9 Voir p. $9(*)$.

10 Cf. Hugo (V.), Op.cit, p. 270.

11 Voir p. 9.

12 Voir p. 14, 15. 